\title{
Comunicação
}

[Communication]

\section{Desvio para a esquerda associado a compartimento medular de reserva dos neutrófilos não esgotado em cadelas portadoras de piometra}

\author{
[Nuclear left deviation associated with medullary reserve compartment of non \\ exhausted neutrophils in female dogs with pyometra] \\ P.R.O. Paes, M.G.M.G. Andrade, F.O.P. Leme, L.B. Veloso, R.D.O. Pereira, \\ C. Malm, M.M. Melo, N.A.M. Silva \\ Escola de Veterinária - UFMG - Belo Horizonte, MG
}

A piometra, que literalmente significa pus no útero, é a doença reprodutiva mais comum em cadelas de meia-idade (Bartoskova et al., 2007). Para a confirmação da enfermidade, a ultrassonografia e o hemograma são os exames complementares mais utilizados (Khan et al., 2008). Neste último, as alterações mais comuns são anemia, leucocitose, neutrofilia, desvio dos neutrófilos para a esquerda, linfopenia e monocitose (Wheathon et al., 1989).

Como na literatura consultada não foi encontrado nenhum relato sobre as possíveis alterações da medula óssea de animais com piometra, objetivou-se, no presente estudo, correlacionar os quadros hematológicos da medula óssea e do sangue periférico de cadelas portadoras dessa enfermidade. Para tal, foram realizados exames hematológicos de medula óssea no momento précirúrgico (M0) e de sangue nos momentos précirúrgico (M0), 24 horas (M24) e 48 horas (M48) após a ovário-histerectomia de 15 cadelas com confirmação clínica de piometra. O projeto de pesquisa foi aprovado pelo Comitê de Ética em Experimentação Animal da UFMG (CETEA), sob o número de protocolo 270/2008.

Para a realização do hemograma, foram coletados $3 \mathrm{~mL}$ de sangue, em tubo, com o anticoagulante etilenodiaminotetracético (EDTA) a $10 \%$ para processamento em aparelho hematológico veterinário (Abacus Vet Jr., Diatron) e avaliação morfológica dos tipos celulares, incluindo o exame diferencial de leucócitos em 200 células, conforme recomendação de Harvey (2001). Para a realização do mielograma, foi coletada amostra da medula óssea do manúbrio do esterno, utilizando-se agulha hipodérmica de 0,6 a $0,7 \mathrm{~mm}$ de diâmetro externo por $25 \mathrm{~mm}$ de comprimento, conectada à seringa de $10 \mathrm{~mL}$. Imediatamente após a coleta, foram confeccionadas lâminas por meio da técnica de squash (Harvey, 2001). As amostras coradas com corante hematológico rápido foram examinadas em microscopia óptica para avaliação morfológica de 500 células hematopoiéticas, conforme Harvey (2001).

Os dados obtidos no presente experimento foram analisados em delineamento em bloco ao acaso, com três tratamentos. Utilizou-se o teste-T de Student para coeficientes de variação grandes (VCM, CHCM, RDW e metamielócitos) e o teste de SNK para coeficientes de variação menores; quando necessário, foram realizadas transformações de dados por raiz quadrada (leucóctios totais, segmentados, monócitos e plaquetas) ou logarítmica (metamielócitos, bastonetes, eosinófilos e linfócitos). $\mathrm{Na}$ persistência da ausência de normalidade, foi utilizado o teste não paramétrico de Friedman (mielócitos). Análises de correlação foram realizadas pelo método de Pearson. Todos os testes estatísticos empregados consideraram a margem de significância de $5 \%$.

Os sinais clínicos mais observados nas cadelas portadoras de piometra foram distensão abdominal $(71,4 \%)$, secreção vulvar $(64,3 \%)$, anorexia $(50,0 \%)$, prostração $(42,9 \%)$, poliúria/polidipsia $(28,6 \%)$ e emese $(21,4 \%)$. Essas alterações ocorreram em proporções semelhantes aos relatos de literatura (Cho et al., 2000; Smith, 2006). Os resultados do hemograma estão apresentados na Tab. 1.

Recebido em 17 de março de 2014

Aceito em 5 de setembro de 2014

E-mail: paulopaes@vet.ufmg.br 
Tabela 1. Média e desvios-padrão dos valores obtidos a partir dos hemogramas de cadelas portadoras de piometra, nos momentos que antecederam à ovário-histerectomia (M0) e 24 horas (M24) e 48 horas (M48) após o procedimento cirúrgico

\begin{tabular}{|c|c|c|c|c|}
\hline \multirow{2}{*}{ Testes } & \multicolumn{3}{|c|}{ Momentos } & \multirow{2}{*}{$\begin{array}{l}\text { Valores de } \\
\text { referência }\end{array}$} \\
\hline & M0 & M24 & M48 & \\
\hline $\mathrm{VG}(\%)$ & $31,67 \pm 6,9 a$ & $31,00 \pm 7,7 \mathrm{a}$ & $31,07 \pm 8,2 \mathrm{a}$ & $37-55$ \\
\hline Hemoglobina (g/dL) & $10,61 \pm 2,7 a$ & $10,75 \pm 3,0 \mathrm{a}$ & $10,56 \pm 3,0$ & $12-18$ \\
\hline Hemácias (x10 céls/ $\mu \mathrm{L})$ & $4,62 \pm 1,1 \mathrm{a}$ & $4,57 \pm 1,3 \mathrm{a}$ & $4,62 \pm 1,4$ & $5,5-8,5$ \\
\hline VCM (fL) & $68,63 \pm 4,2 \mathrm{a}$ & $68,46 \pm 4,4 a$ & $68,33 \pm 5,5 a$ & $60-77$ \\
\hline $\mathrm{CHCM}(\mathrm{g} / \mathrm{dL})$ & $33,17 \pm 1,5 \mathrm{a}$ & $34,47 \pm 1,8 \mathrm{a}$ & $33,68 \pm 1,5 \mathrm{a}$ & $31-37$ \\
\hline RDW $(\%)$ & $14,07 \pm 0,9 \mathrm{a}$ & $14,03 \pm 0,9 a$ & $14,20 \pm 0,9 \mathrm{a}$ & $12-15$ \\
\hline \multirow[t]{2}{*}{ Leucócitos totais $(/ \mu \mathrm{L})$} & 27.043 & 57.940 & 40.139 & $6.000-17.000$ \\
\hline & $\pm 15.937 \mathrm{a}$ & $\pm 22.868 \mathrm{c}$ & $\pm 18.724 \mathrm{~b}$ & \\
\hline Mielócitos $(/ \mu \mathrm{L})$ & $326 \pm 1.089 \mathrm{a}$ & $192 \pm 745 a$ & $26 \pm 102 \mathrm{a}$ & 0 \\
\hline Metamielócitos $(/ \mu \mathrm{L})$ & $835 \pm 1.813 \mathrm{a}$ & $615 \pm 1.243 \mathrm{a}$ & $589 \pm 860 \mathrm{a}$ & 0 \\
\hline Bastonetes $(/ \mu \mathrm{L})$ & $4.893 \pm 4.098 \mathrm{a}$ & $5.366 \pm 5.259 \mathrm{a}$ & $3.669 \pm 4.143 \mathrm{a}$ & $0-300$ \\
\hline \multirow[t]{2}{*}{ Segmentados $(/ \mu \mathrm{L})$} & 15.849 & 43.444 & 31.288 & $3.000-11.500$ \\
\hline & $\pm 10.025 \mathrm{a}$ & $\pm 20.340 \mathrm{c}$ & $\pm 17.006 \mathrm{~b}$ & \\
\hline Eosinófilos $(/ \mu \mathrm{L})$ & $199 \pm 347 \mathrm{a}$ & $442 \pm 1.236 \mathrm{a}$ & $191 \pm 241 \mathrm{a}$ & $100-1.250$ \\
\hline Linfócitos $(/ \mu \mathrm{L})$ & $2.617 \pm 1.688 \mathrm{a}$ & $4.003 \pm 2.839 \mathrm{a}$ & $2.078 \pm 1.566 \mathrm{a}$ & $1.000-4.800$ \\
\hline Monócitos $(/ \mu \mathrm{L})$ & $2.188 \pm 1.932 \mathrm{a}$ & $3.633 \pm 2.189 \mathrm{a}$ & $2.298 \pm 1.081 \mathrm{a}$ & $150-1.350$ \\
\hline Plaquetas $\left(\mathrm{x} 10^{3} / \mu \mathrm{L}\right)$ & $209,40 \pm 92 \mathrm{a}$ & $260,40 \pm 143 a$ & $278,53 \pm 132 \mathrm{a}$ & $175-500$ \\
\hline
\end{tabular}

No eritrograma (Tab. 1), os valores médios foram compatíveis com anemia discreta, normocítica normocrômica, com $80,0 \%$ das cadelas apresentando anemia, sendo metade classificada, conforme Tvedten (2006), como discreta (VG:30-36\%) e outra metade como moderada (VG:20-29\%). Não foram observadas alterações compatíveis com anemia responsiva, como presença de macrocitose, hipocromia, anisocitose, policromasia ou corpúsculos de Howell-Jolly. Esses achados, associados aos resultados da série eritroide da medula óssea (Tab. 2), que incluem alta relação mieloide:eritroide (ME), baixa concentração de células precursoras eritroides tardias (rubrícitos e metarrubrícitos) e alta concentração de reservas de ferro, permitem associar os resultados obtidos à anemia da doença inflamatória que, segundo Thrall (2004), são caracterizados pela indisponibilidade do ferro medular, aumento da destruição de hemácias e diminuições da produção de eritropoietina nos rins e no fígado, assim como da resposta a este hormônio na medula óssea.

No M0, a média de leucócitos totais (Tab.1) e a porcentagem de animais com leucocitose
$(73,3 \%)$, leucopenia $(6,7 \%)$ e contagem de leucócitos acima de 30.000 céls/ $\mu \mathrm{L}$ (33,3\%) estão próximas aos descritos na literatura para cadelas portadoras da enfermidade (Schepper $e t$ al., 1987; Wheathon et al., 1989; Sevelius et al., 1990; Plavec et al., 2006; Bartoskova et al., 2007; Khan et al., 2007). Outros achados relevantes foram desvio para a esquerda dos neutrófilos (80,0\%), linfopenia (20,0\%), eosinopenia $(46,7 \%)$ e monocitose $(46,7 \%)$. Segundo Smith (2000) e Schultze (2000), a neutrofilia ocorre devido à produção e liberação de neutrófilos superar a demanda tecidual, ao passo que a linfopenia, a eosinopenia e a monocitose são atribuídas principalmente à ação dos glicocorticoides endógenos.

No M24, a contagem de leucócitos totais e a porcentagem de animais com contagem acima de 30.000 céls $/ \mu \mathrm{L}(93,3 \%)$ foram bastante superiores quando comparadas aos momentos pré-cirúrgicos da piometra, tanto do presente experimento, quanto dos relatos de literatura. Essa elevação da leucometria se deve, de acordo com Gaunt (2000), à retirada do foco inflamatório. No M48, a permanência de valores alterados no leucograma sugere a persistência, embora 
em menor concentração do que em M24, de mediadores inflamatórios, possivelmente liberados em razão da presença de toxinas bacterianas, já que o tempo de permanência médio dos leucócitos na circulação é de poucas horas. Provavelmente a leucocitose no M48 também foi influenciada pelo evento cirúrgico. Os resultados médios das concentrações de leucócitos no M0 e no M48 (27.043 céls/ $\mu \mathrm{L}$ e 40.139 céls $/ \mu \mathrm{L}$, respectivamente) são muito próximos aos relatados por Fransson (2003), em experimento e momentos semelhantes (27.000 céls $/ \mu \mathrm{L}$ e 45.300 céls $\mu \mathrm{L}$, respectivamente). Segundo Plavec et al. (2006) e Bartoskova et al. (2007), a contagem de leucócitos tende à normalização após uma semana da ováriohisterectomia.

Tabela 2. Valores médios e desvios-padrão de mielogramas de cadelas portadoras de piometra, no momento que antecedeu à ováriohisterectomia (M0)

\begin{tabular}{|c|c|c|}
\hline Testes & $\begin{array}{l}\text { Momento } \\
\text { M0 }\end{array}$ & $\begin{array}{l}\text { Valores de } \\
\text { referência }\end{array}$ \\
\hline Celularidade $(\%)$ & $77,1 \pm 9,7$ & $25-75$ \\
\hline Relação M:E & $26,3 \pm 21,3$ & $0,75-2,53$ \\
\hline Ferro & $2,0 \pm 0,8$ & $0 *$ \\
\hline $\begin{array}{l}\text { Megacariócitos } \\
\text { (/partícula) }\end{array}$ & $6,7 \pm 6,8$ & $2-7$ \\
\hline Mieloblastos (\%) & $2,01 \pm 2,6$ & $0,4-1,1$ \\
\hline Promielócitos (\%) & $5,19 \pm 2,3$ & $1,1-2,3$ \\
\hline Mielócitos (\%) & $9,08 \pm 2,6$ & $3,1-6,1$ \\
\hline $\begin{array}{l}\text { Metamielócitos } \\
(\%)\end{array}$ & $14,66 \pm 3,4$ & $5,3-8,8$ \\
\hline Bastonetes (\%) & $33,49 \pm 7,7$ & $12,7-17,2$ \\
\hline Segmentados (\%) & $19,82 \pm 8,7$ & $13,8-24,2$ \\
\hline Eosinófilos (\%) & $1,23 \pm 1,1$ & $1,8-5,6$ \\
\hline Basófilos (\%) & $0,04 \pm 0,1$ & $0-0,8$ \\
\hline Linfócitos (\%) & $3,08 \pm 2,8$ & $1,7-4,9$ \\
\hline Plasmócitos (\%) & $1,66 \pm 1,5$ & $0,6-2,4$ \\
\hline Monócitos (\%) & $1,01 \pm 1,0$ & $0,4-2,4$ \\
\hline Rubriblastos (\%) & $1,30 \pm 1,3$ & $0,2-1,1$ \\
\hline Prorrubrícitos (\%) & $1,23 \pm 1,4$ & $0,9-2,2$ \\
\hline Rubrícitos (\%) & $4,27 \pm 7,6$ & $19,2-35,1$ \\
\hline Metarrubrícitos & $1,92 \pm 2,9$ & $9,2-16,4$ \\
\hline
\end{tabular}

*Concentração de ferro: (0) normal, (1) discretamente aumentada, (2) moderadamente aumentada, (3) intensamente aumentada. Relação M:E (relação mieloide:eritroide).

No exame da medula óssea, a concentração celular (celularidade) e a relação ME elevadas
(Tab. 2) indicam o direcionamento da medula óssea para a produção de células de defesa e, dessa forma, são compatíveis com a leucocitose observada no sangue. Entretanto, o desvio para a esquerda dos neutrófilos do sangue periférico (Tab. 1), normalmente associado, segundo Smith (2000), ao esgotamento do compartimento de reserva medular de neutrófilos, ocorreu majoritariamente associado a estoques medulares normais ou aumentados de neutrófilos segmentados $\quad(50 \%$ e $\quad 33 \%$ dos animais com desvio para a esquerda, respectivamente), incluindo os casos de desvio para a esquerda degenerativo $(13,3 \%$ dos animais no M0). Esse achado, associado à ausência de correlação entre o compartimento de reserva da medula óssea e a contagem de bastonetes do sangue, indica que, diferentemente do citado em literatura, o desvio para a esquerda não está necessariamente associado ao esgotamento do estoque de segmentados na medula óssea, e sim à desproporcionalidade entre as concentrações celulares dos compartimentos de proliferação (mieloblastos, promielócitos e mielócitos) e maturação (metamielócitos e bastonetes) com o compartimento de reserva (segmentados) dessa linhagem celular.

A presença de megacariócitos em concentração média próxima ao limite superior de referência (Tab. 2), concomitante à contagem média de plaquetas do sangue próxima ao limite inferior de referência (Tab. 1), sugere consumo e/ou destruição aumentados de plaquetas. Entretanto, permanece não esclarecido o fato dos dois animais $(13,3 \%)$ que apresentaram concentrações de megacariócitos acima do dobro do limite de referência (>14/partícula) serem os mesmos em que a relação $\mathrm{ME}$ encontrava-se dentro dos valores de referência.

Conclui-se que o desvio para a esquerda na piometra de cadelas está principalmente associado à desproporcionalidade entre as concentrações celulares dos compartimentos de proliferação e maturação dos neutrófilos com o compartimento de reserva dessa linhagem celular e não ao esgotamento do estoque de segmentados na medula óssea.

Palavras-chave: piometra, hematologia, medula óssea, desvio para a esquerda, mielograma 


\begin{abstract}
From fifteen female dogs with clinical diagnoses of pyometra, hematological exams were performed at three times: MO (prior to the surgery), M24 (24h after ovarysalpingohisterectomy-OSH) and M48 (48h after OSH). Anemia was seen in $80 \%$ of the cases, characterized as mild normocytic normochromic type. The means of total leukocyte counts were 27.043, 57.940 and 40.139 céls/ $\mu$ L in M0, M24 and M48. A total of $80 \%$ of the animals presented neutrophilic left shift in all moments. During medullar exams, the cellular, iron reserve and megakaryocytic concentration were raised as well as the ME ratio, showing a value of 26,3:1,0, probably due to the elevation of granular proliferation and maturation compartment, as the mean of the reserve compartment was within normal range. As $83 \%$ of the animals with neutrophilic left shift showed a melullary reserve compartment raised, it can be concluded that female dogs with piometra had left shift of neutrophils with disproportionally between compartments, without segmented medullar saturation.
\end{abstract}

Keywords: pyometra, hematology, bone marrow, left shift, myelogram

\section{REFERÊNCIAS}

BARTOSKOVA, A.; VITASEK, R.; LEVA, L.; FALDINA, M. Hysterectomy leads to fast improvement of haematological and immunological parameters in bitches with pyometra. J. Small Anim. Pract., v.48, p.564$568,2007$.

CHO, J.; KIM, H.; LEE, S. et al. Clinical study of canine pyometra. Korean J. Vet. Clin. Med. v.17, p.219-224, 2000.

FRANSSON, B.A. Systemic inflammatory response in canine pyometra. 49p. 2003. Tese (doutorado).Uppsala: Swedish University of Agricultural Science,

GAUNT, S.D. Extreme neutrophilic leukocytosis In: FELDMAN, B.; ZINKL, J.; JAIN, N.C. Schalm's Veterinary Hematology. 5.Ed., Philadelphia: Lippincott Williams \& Wilkins. Cap.52, 2000. p.347-349.

HARVEY, J.W. Atlas of Veterinary Hematology. Philadelphia: Saunders Company, 2001. 228p.

KHAN, L.A.; RAGHUWANSHI, D.S.; VHORA, S.C. et al. Comparative efficacy of different methods for diagnosis of pyometra. Royal Vet. J. India. v.4, p.43-45, 2008.

PLAVEC, T.; CELINJEK, B.; DOLINAK, K. et al. Haemostasis impairment in bitches with pyometra. Acta Vet. (Beograd), v.56, p.529-530, 2006.
SCHEPPER, J.; STOCK, J.; CAPIAU, E. Anaemia and leucocytosis in one hundred and twelve dogs with pyometra. J.small anim.pratic. v.28, p.137-145, 1987.

SCHULTZE, A.E. Interpretation of canine leukocyte responses In: FELDMAN, B.; ZINKL, J.; JAIN, N.C. Schalm's veterinary hematology. 5 Ed., Philadelphia: Lippincott Williams \& Wilkins. Cap.55, p.366-381, 2000.

SEVELIUS, E.; TIDHLOLM, A.; THORENTOLLING, K. Pyometra in the dog. J.Am.Anim.Hosp.Assoc. v.26, p.33-38, 1990.

SMITH, G.S. Neutrophils. In: FELDMAN, B.; ZINKL, J.; JAIN, N.C. Schalm's veterinary hematology. 5.Ed., Philadelphia: Lippincott Williams \& Wilkins. Cap.46, p.281-296, 2000.

SMITH, F.O. Canine pyometra. Theriogenology, v.66, p.610-612, 2006.

THRALL, M.A. Veterinary hematology and clinical chemistry. Philadelphia: Lippincott Williams \& Wilkins, 2004. 518p.

TVEDTEN, H. Laboratory and clinical diagnosis of anemia. In: WEISS D.J.; WARDROP, K. J. Schalm's veterinary hematology. 6. ed. Iowa: Wiley -Plackwell. Cap.24, 2006. p.152-161.

WHEATON, L.G.; JOHNSON, A.L.; PARKER, A.J.; KNELLER, S.K. Results and complications of surgical treatment of pyometra: a review of 80 cases. J. Am. Anim. Hosp. Assoc., v.25, p.563$568,1989$. 